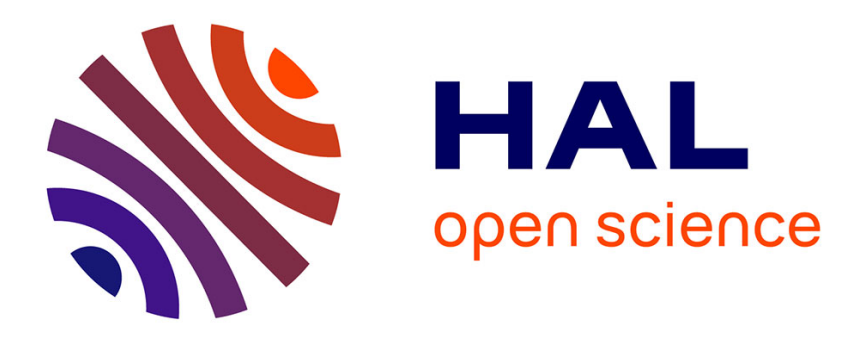

\title{
Pour une approche géographique de la convergence économique
}

Bourdin Sébastien

\section{To cite this version:}

Bourdin Sébastien. Pour une approche géographique de la convergence économique: L'exemple des inégalités régionales et leurs évolutions dans l'Union Européenne. Espace Géographique, 2013, 42, 10.3917/eg.423.0270 . hal-02066343

HAL Id: hal-02066343

https://hal-normandie-univ.archives-ouvertes.fr/hal-02066343

Submitted on 13 Mar 2019

HAL is a multi-disciplinary open access archive for the deposit and dissemination of scientific research documents, whether they are published or not. The documents may come from teaching and research institutions in France or abroad, or from public or private research centers.
L'archive ouverte pluridisciplinaire HAL, est destinée au dépôt et à la diffusion de documents scientifiques de niveau recherche, publiés ou non, émanant des établissements d'enseignement et de recherche français ou étrangers, des laboratoires publics ou privés. 


\title{
POUR UNE APPROCHE GEOGRAPHIQUE DE LA CONVERGENCE ECONOMIQUE \\ L'EXEMPLE DES INEGALITES REGIONALES ET LEURS EVOLUTIONS DANS L'UNION EUROPEENNE
}

\section{FOR A GEOGRAPHICAL APPROACH OF THE ECONOMIC CONVERGENCE THE EXAMPLE OF THE REGIONAL DISPARITIES AND THEIR EVOLUTIONS IN THE EUROPEAN UNION}

\author{
BOURDIN Sébastien \\ Institut du Développement Territorial \\ 9 Rue Claude Bloch 14052 Caen \\ bourdin.sebastien@gmail.com
}

Résumé

L'objectif de cet article est de poser les fondements théoriques et méthodologiques sur la convergence économique. La prise en compte de la dimension spatiale de la mesure de la convergence constitue de ce point de vue une perspective intéressante dans le champ de la géographie. L'indice de convergence économique locale proposé permet de mesurer les processus simultanés de convergence et de divergence économique qui s'opèrent dans l'Union européenne. En comparant les deux périodes 1995-2000 et 20002006 sur l'ensemble des régions européennes au niveau NUTS 2-3, il apparaît des situations de régions qui ont évolué de manière favorable ou défavorable d'un point de vue économique. La structuration spatiale de ces phénomènes de convergence et de divergence économique locale est très marquée et traduit une véritable géographie de l'évolution des inégalités régionales dans l’Union européenne.

Mots-clés : convergence - analyse spatiale - croissance régionale - disparités régionales - Union européenne

\section{Summary}

The aim of this paper is to lay the theoretical and methodological foundations on the convergence. Taking into account the spatial dimension of the measure of convergence is from this point of view an interesting perspective in the field of the geography. The index of local convergence proposed enable to measure the simultaneous processes of economic convergence and divergence occurring in the European Union.

Key-words : convergence - spatial analysis - regional disparities - regional growth - European Union 


\section{Introduction}

En 2010, une étude d'Eurostat montre que la région Inner London (ville centre de Londres) est la plus prospère de l'Union européenne (UE) et est sept fois plus riche que la région la moins développée à savoir la région Severozapaden située en Bulgarie. Des études antérieures (EUROSTAT, 2007 et 2009) insistent sur le caractère très prononcé des inégalités économiques régionales entre l'ex-Europe de Quinze et les régions des pays d'Europe centrale et orientale (PECO). Alors que l'élargissement de l'UE aux dix nouveaux membres des PECO est désormais acquis, la question de la convergence économique ${ }^{1}$ des régions et du devenir de la politique de cohésion est posée.

La réduction des disparités régionales est une condition de la réussite du marché non plus commun mais unique. La convergence des niveaux de vie doit permettre de garantir une concurrence loyale à l'intérieur de l'espace européen. Ainsi, il n'est pas étonnant de constater que la grande réforme de la politique régionale de 1988 correspond précisément avec la volonté d'achèvement du marché unique (Acte unique, 1986). Les grands Objectifs de la politique de cohésion ont connu des évolutions, mais le principe n'a pas changé. On cherche surtout aujourd'hui à ne plus saupoudrer les aides mais, au contraire, à les concentrer sur les régions les plus en retard. Certains ont même prôné une suppression de «l'interventionnisme » européen et une renationalisation des aides (Sapir, 2003).

Aujourd'hui, les douze nouveaux États-membres disposent de 53\% des Fonds structurels mais des aides transitoires sont accordées aux régions brusquement enrichies en moyenne par l'arrivée de régions en grande difficulté. L'élargissement à dix pays - dont le PIB moyen par habitant est inférieur à $43 \%$ de celui de l'Europe des Quinze - remet en cause les équilibres budgétaires sur lesquels repose le financement de la politique régionale européenne. Les vifs débats actuels sur le futur budget de l'Union européenne pour la période 2014-2020 montrent que l'enjeu est aussi bien économique que politique. Il réside dans le maintien même de la politique de cohésion ou sa révision.

De nombreuses publications (rapports européens, articles scientifiques) traitent de la question de l'évaluation du processus de convergence économique dans l'UE. Pourtant, dans le cas d'une grande partie des rapports européens, la prise en compte de l'espace géographique dans l'explication de la convergence est rarement prise en compte. En revanche, on observe de plus en plus dans la littérature scientifique en économie une mise en évidence des « effets de débordement géographique » (autrement dit des effets de voisinage en géographie) dans l'explication de la croissance régionale et la convergence économique.

\footnotetext{
${ }^{1}$ Catherine Fuss (1999) estime qu'il y a « convergence lorsque la différence entre des séries ou leur dispersion se réduit dans le temps ».
} 
L'objectif de cet article est donc d'appréhender la convergence économique d'un point de vue géographique. Nous verrons donc dans quelle mesure l'approche spatiale du processus de convergence permet d'avoir une nouvelle lecture des disparités économiques régionales et de l'intégration régionale.

Dans un premier temps, nous synthétiserons le cadre théorique et méthodologique sur la convergence économique puis nous présenterons dans la lignée de ce contexte une mesure locale de la convergence prenant en compte l'espace géographique. La seconde partie quant à elle consistera à analyser et expliquer les trajectoires régionales de convergence identifiées.

\section{Vers une approche spatiale de la convergence économique}

L'objectif de cette partie est de présenter comment les travaux sur la convergence économique sont passés d'une approche purement économique globale (via notamment les tests de bêta et de sigma convergence) à une approche locale prenant en compte l'espace géographique. C'est dans ce contexte qu'un indice original de convergence locale est proposé pour évaluer l'évolution des disparités régionales par une approche géographique.

\subsection{De la convergence " globale " à la convergence locale : fondements théoriques et méthodologiques}

A l'origine des travaux sur la convergence économique on retrouve la théorie néo-classique de la croissance développée par Robert Solow (1956) selon laquelle la convergence économique entre pays inégalement développés est possible. Il part du principe qu'il existe des disparités régionales initiales et qu'elles tendent à se résorber avec le temps. Pour cela, chaque région converge vers un taux de croissance du revenu par tête de long terme appelé le «taux d'état stationnaire $»^{2}$. Ainsi, une économie aura tendance à converger plus rapidement si elle se situe en dessous de son état stationnaire.

Durant cette dernière décennie, les études empiriques sur la convergence économique ont connu un essor important et présentent souvent des résultats contradictoires (Henin, Le Pen, 1995 ; Quah, Durlauf, 1998 ; Islam, 2003 ; Abreu et al., 2005 ; Dall'erba, Le Gallo, 2008 ; Le Pen, 2011). L'origine de la sensibilité de ces résultats peut être recherchée dans les différences de conceptions de la convergence et donc des méthodologies inhérentes à chacune de ces approches.

Parmi elles, on retrouve les tests de convergence en coupe transversale introduits par William Baumol (1986), puis développés par Robert J. Barro, Xavier Sala-i-Martin (1991 et 1992). Ils se décomposent en deux tests (bêta et sigma convergence). La $\beta$-convergence s'attache à mettre en évidence un éventuel rattrapage des pays riches par les pays pauvres (relation négative entre le taux de variation et le niveau initial) tandis que la $\sigma$-convergence cherche à mesurer l'évolution des disparités économiques (réduction des écarts de PIB/hab. entre deux dates).

\footnotetext{
${ }^{2}$ L'état stationnaire est un phénomène important à deux égards : une économie qui l'a atteint ne bouge plus et une économie qui ne l'a pas atteint tend vers lui. L'état stationnaire représente l'équilibre de longue période de l'économie.
} 
D'une part, il existe l'approche par la $\beta$-convergence. Dans cette situation, l'hypothèse de convergence implique que le pays/la région qui initialement a un PIB/hab. plus faible devrait connaitre un taux de croissance plus rapide durant la période de rattrapage. Elle peut être absolue (inconditionnelle) ou conditionnelle. Elle est absolue lorsqu'elle est indépendante des conditions initiales. Elle cherche alors à évaluer si les niveaux de vie des économies développées tendent à être rattrapés par les niveaux de vie des économies moins développées. Elle est conditionnelle lorsque les variables d'état des économies régionales considérées sont supposées similaires en termes de capital humain, physique ou encore de taux de croissance de la population (Mankiw et al., 1992). Ces facteurs dits «endogènes » déterminent les conditions initiales et la convergence des économies vers un état stationnaire dépend de celles-ci.

D'autre part, la $\sigma$-convergence s'attache à observer si la dispersion de la richesse se réduit au cours du temps. L'idée est de vérifier si les PIB/hab. se rapprochent du niveau moyen des pays ou régions observés. Si les écarts à la moyenne se réduisent au cours de la période étudiée, on dit alors qu'il y a $\sigma$-convergence. Il s'agit donc de comparer un indicateur de dispersion, calculé pour la fin de la période, à cet indicateur calculé pour le début de la période. Il existe plusieurs indicateurs de mesure de la dispersion (coefficient de variation, écart-type, coefficient de Gini, d'indice d'Atkinson ou de Theil, écarts à la moyenne logarithmique) avec chacun des propriétés spécifiques de focalisation sur des niveaux particuliers d'inégalités économiques (Montfort, 2008).

Les tests économétriques et statistiques de bêta et sigma convergence réalisés sur un large échantillon de pays et sur près de trente ans conduisent à rejeter l'hypothèse de rattrapage. En d'autres termes, la croissance des pays initialement les moins développés n'a pas été systématiquement plus rapide que celle des pays développés. A la suite de ces études, de nouveaux travaux sur la convergence économique ont émergé avec le courant de la Nouvelle Économie Géographique (NEG) qui s'est développé dans les années 90 avec Paul Krugman (1991). Il part du constat suivant : les activités économiques sont concentrées autour de pôles (appelés aussi « clusters ») et cette polarisation modifie la répartition spatiale des richesses entre les régions. Les travaux précurseurs de Jeffrey G. Williamson (1965) sont à l'origine de la naissance de ce courant économique puisque déjà, à l'époque, il mettait en évidence le rôle de l'espace pour expliquer la croissance régionale. Le rôle de l'espace dans cette théorie économique est majeur car il contribue à expliquer les phénomènes de croissance économique. La répartition géographique des phénomènes de croissance à l'échelle régionale est donc rarement aléatoire: les performances économiques de régions voisines sont, au contraire, souvent similaires (Getis, 1991).

Les études sur la convergence issues de la NEG montrent que certains pays arrivent davantage à tirer partie de la croissance tandis que d'autres échouent dans cette tentative d'en profiter. On observe alors à la fois la convergence économique de certains et la divergence d'autres dans un mouvement synchrone. Le résultat de ce processus n'est pas déterminé à l'avance et les nouveaux modèles théoriques mettant en avant le rôle de l'espace géographique montrent que l'on peut produire à la fois de la convergence et de la divergence 
économique. Par conséquent, les effets de la convergence économique sur l'intégration régionale peuvent être à la fois positifs et négatifs. Cela dépend donc beaucoup de la situation initiale, de la capacité des régions à s'adapter et des effets de voisinage. C'est dans ce cadre que cet article propose une mesure de la convergence locale pour évaluer ces phénomènes simultanés de convergence et de divergence économique.

\subsection{La convergence locale : émergence d'un concept et nouvelles méthodes de mesure}

L'intérêt d'une mesure de la convergence économique au niveau local réside dans le fait qu'elle permettra de comprendre l'origine de la persistance ou au contraire de la réduction des disparités régionales dans certaines parties de l'UE. Il est possible de définir la convergence locale comme étant une situation où une convergence s'observe entre des territoires proches localisés dans l'espace. L'intérêt est donc de prendre en compte non plus seulement les conditions économiques mais aussi les proximités géographiques comme pouvant potentiellement influencer la convergence économique. Par exemple, l'absence de rattrapage (voire la divergence) d'une région en retard s'expliquerait par le fait qu'elle soit entourée d'autres régions en retard.

La convergence locale mesure ici la réduction (ou l'augmentation) des disparités économiques au sein d'un groupe de régions voisines. Autrement dit, il y a convergence locale quand les $\mathrm{PIB} / \mathrm{hab}$. des régions comprises dans la zone délimitée se rapprochent du niveau moyen des PIB/hab. de la zone considérée.

\section{Encadré 1 : Le PIB/hab. : un indicateur possible pour mesurer la convergence économique}

Dans les études menées sur la convergence économique des régions et des pays, le $\mathrm{PIB} / \mathrm{hab}$. (sous ses différentes formes) est l'indicateur le plus souvent utilisé. Bien qu'il existe des aspects qui limitent la portée de cet indicateur - pour des raisons de mesure (du fait de la production domestique, de l'économie souterraine, des migrations) ou d'interprétation (en raison des inégalités) - le PIB reste l'indicateur privilégié quand il s'agit de mener des comparaisons entre pays et entre régions. Depuis 2007, un groupe d'experts de la Commission européenne « Beyond GDP » tente cependant de dépasser cet indicateur en proposant des mesures alternatives. En économie de marché, le PIB/hab. constitue l'outil principal de mesure des performances économiques. II est aussi l'indicateur utilisé par l'UE pour l'attribution des Fonds structurels : un seuil de $75 \%$ du PIB/hab. communautaire moyen définit les régions défavorisées éligibles à l'Objectif 1 . De fait, lorsque l'on souhaite à la fois étudier le processus de convergence économique et le rôle joué par les Fonds structurels, il semble incontournable de prendre le PIB/hab. comme variable d'analyse. En outre, disposer d'une mesure des valeurs ajoutées régionales est utile dans le cadre d'une analyse de la différenciation économique et spatiale régionale : elle permet d'effectuer des comparaisons quantitatives entre les régions de l'UE. Aussi, afin de corriger les distorsions monétaires des PIB nationaux, une évaluation en parité de pouvoir d'achat (PPA) est utilisée. Elle reflète de façon plus exacte les pouvoirs d'achat intérieurs et les niveaux de développement.

Nous avons formalisé cet indice de convergence locale comme suit :

$$
C_{i}=\sum_{j \in \mathrm{V}(i)} \frac{\frac{\sigma_{n}^{j}}{m_{n}^{j}}-\frac{\sigma_{0}^{j}}{m_{0}^{j}}}{n}
$$

$C_{i}$ mesure l'évolution de la dispersion du $\mathrm{PIB} /$ hab. sur $n$ années pour une région $i$ et ses voisines $j$. On calcule l'écart annuel du coefficient de variation entre deux périodes $t_{0}$ et $t_{n}$ pour V( $\left.i\right)$. 
$m_{n}^{j}$ est le PIB/hab moyen de l'année $n$ de $\mathrm{V}(i)$ et $\sigma_{n}^{j}$ son écart-type.

$\mathrm{V}(\mathrm{i})$ est composé de la région i et de l'ensemble des régions à moins de $\delta$ de la région $\mathrm{i}$

$\delta$ est la distance maximale (en $\mathrm{km}$ ) définissant le périmètre $\mathrm{V}(\mathrm{i})$ entre une région $i$ et ses voisines $j$. Il s'agit d'une fonction de distance à seuil fixe

$t$ est la différence de temps entre l'année 0 et l'année $n$

Arthur Getis (2009) et Richard Rogerson (2010) ont tous les deux récemment montré qu'il n'existait pas en l'état de solution pour définir une méthode de voisinage optimale. Nous gardons une préférence pour la prise en compte de la distance à vol d'oiseau comme mode de définition du voisinage car la distance euclidienne simule une situation théorique d'homogénéité des réseaux de transports et d'égalité face à l'accessibilité. Une des limites de ce choix réside dans le fait que tous les voisins sont égaux, quelque soit leur éloignement réel. Une approche gaussienne de la distance permettrait de corriger cette limite. Une autre méthode envisageable consisterait à prendre en compte la topologie des limites régionales (méthode des «plus proches voisins»). Même si elle permet de maitriser le nombre de voisins, elle ne prend cependant pas en compte l'éloignement des territoires. Des matrices prenant en compte la distance-temps (Virol, 2006) ou l'accessibilité par la route (Toral, 2002 ; Dall'Erba, 2004) auraient été idéales mais la disponibilité des données ne nous permettait pas de les utiliser pour toute l'Union européenne.

Afin d'évaluer le niveau de significativité de l'indice de convergence locale, une inférence statistique sur les résultats obtenus a été effectuée avec le test non-paramétrique de Kolmogorov-Smirnov. Il apparait que les valeurs pour chaque région de l'indice de convergence locale sont significativement différentes de zéro (avec une valeur critique de $\mathrm{p}=0,05$ - tableau 1 ).

La mesure de la $\sigma$-convergence au niveau local proposée ici permet d'identifier un éventuel phénomène de convergence ou de divergence économique d'une région vis-à-vis de ses voisines. D'autres contributions récentes sur la convergence locale ont été publiées cette dernière décennie. Elles introduisent également la localisation dans l'analyse de la convergence économique (Le Gallo, 2004) et permettent ainsi d'évaluer l'hétérogénéité spatiale et l'autocorrélation spatiale locale de ce processus. Ces méthodes utilisent des modèles de $\beta$-convergence (conditionnelle) localisée afin d'étudier un éventuel rattrapage des régions vers un état-stationnaire.

Les approches proposées par les économistes évaluent la $\beta$-convergence au niveau local en intégrant soit la dimension spatiale des données (Fingleton, Lopez-Bazo, 2006 ; Ertur, Koch, 2007 ; Dall'Erba, Le Gallo, 2008) soit les interdépendances spatiales (Abreu et al., 2005 ; Janikas, Rey, 2005 et 2008 ; Arbia, 2006 ; Rey, Le Gallo, 2009 ; Ertur, Le Gallo, 2009). Une première méthode consiste à utiliser la Régression Géographiquement Pondérée (GWR - Geographically Weighted Regression) afin d'estimer localement la qualité de la régression et les paramètres du modèle de $\beta$-convergence (Bivand, Brunstad, 2003 et 2005 ; Yu, 2006 ; Eckey et al., 2007 ; Yildrim et al., 2009). Il s'agit alors d'estimer un modèle de convergence conditionnelle prenant en compte le décalage spatial. La seconde méthode permet également d'évaluer la 
variation spatiale des taux de croissance. Elle est basée sur une approche de type bayésienne spatiale (BSALE - Bayesian Spatial Autoregressive Local Estimation). Elle permet de prendre en compte à la fois l'hétérogénéité spatiale et l'autocorrélation spatiale du coefficient de $\beta$-convergence et donc d'intégrer la distance comme facteur explicatif des trajectoires de croissance (Ertur et al., 2007 ; Le Gallo et al., 2011).

Ainsi, ces travaux sur la $\beta$-convergence localisée s'attachent à mesurer l'éventuel rattrapage de régions riches par des régions en retard en introduisant un décalage spatial tandis que la $\sigma$-convergence locale proposée dans cet article cherche à mesurer l'éventuelle réduction des disparités entre deux dates pour des régions voisines dans un périmètre défini. Ces deux approches sont donc complémentaires en mesurant des phénomènes différents de la convergence économique.

\section{De la convergence locale aux transitions locales}

Une analyse diachronique a été menée (carte 1) et permet de mettre en lumière des transitions locales de la convergence et de la divergence économique (carte 3). L'idée consiste à identifier les régions dont les trajectoires ont évolué (ou non) entre les deux périodes (et dont les indices sont significatifs). Il apparaît alors des situations de régions qui ont évolué de manière favorable ou défavorable en comparant les deux périodes 1995-2000 et 2000-2006. Il est ainsi possible de montrer au moins en partie les réussites ou échecs de la politique de convergence sur l'ensemble de l'Union européenne.

Dans le cadre de l'étude, le choix a été fait de prendre une portée spatiale de $300 \mathrm{~km}$. Cette dernière correspond à une distance moyenne à l'échelle de l'Union européenne nécessitant moins de 5 heures de voyage en voiture, sur la base des travaux réalisés sur l'accessibilité dans le rapport du Parlement européen (2007). Le rapport précise que le rayon de 5 heures - soit $300 \mathrm{~km}$ - utilisé pour la mesure des situations régionales au niveau local est intéressant lorsqu'il s'agit d'obtenir un aperçu global de la configuration des disparités régionales. Par ailleurs, cette portée permet de mieux appréhender et identifier des zones de convergence et de divergence économique locale que si elle était plus faible (exemple 100km). 
Carte 1: Convergences et divergences locales dans l'UE (voisinage de 300km)

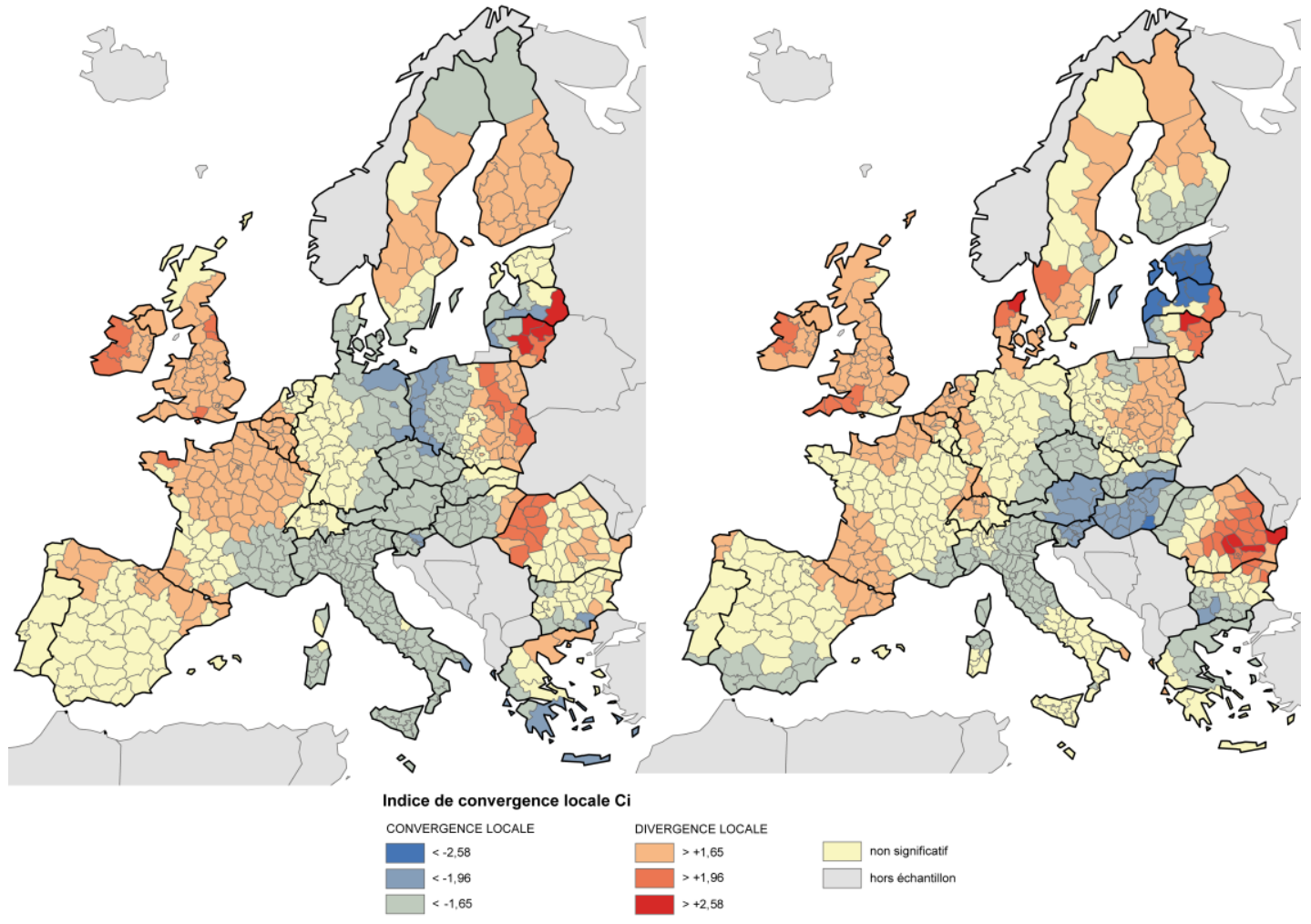

Carte 2 : Les inégalités économiques régionales dans l'Union européenne

LES INÉGALITÉS RÉGIONALES DANS L'UNION EUROPÉENE
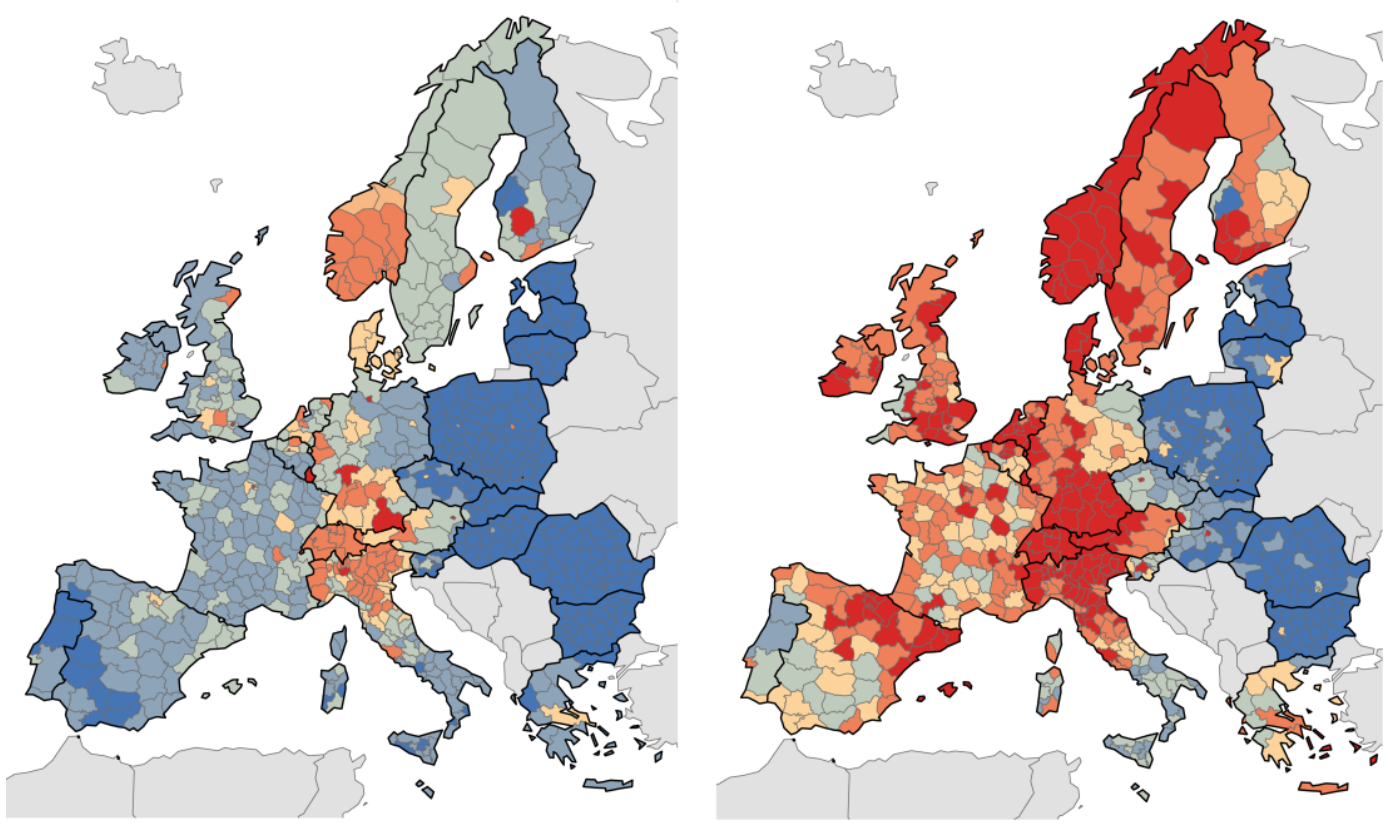

Indice UE27 en $2006=100$

\begin{tabular}{l|l|l}
\hline inf. à 50 & entre 90 à 100 \\
entre 50 et 75 & entre 100 à 125 \\
entre 75 et 90 & sup. à 125
\end{tabular} 

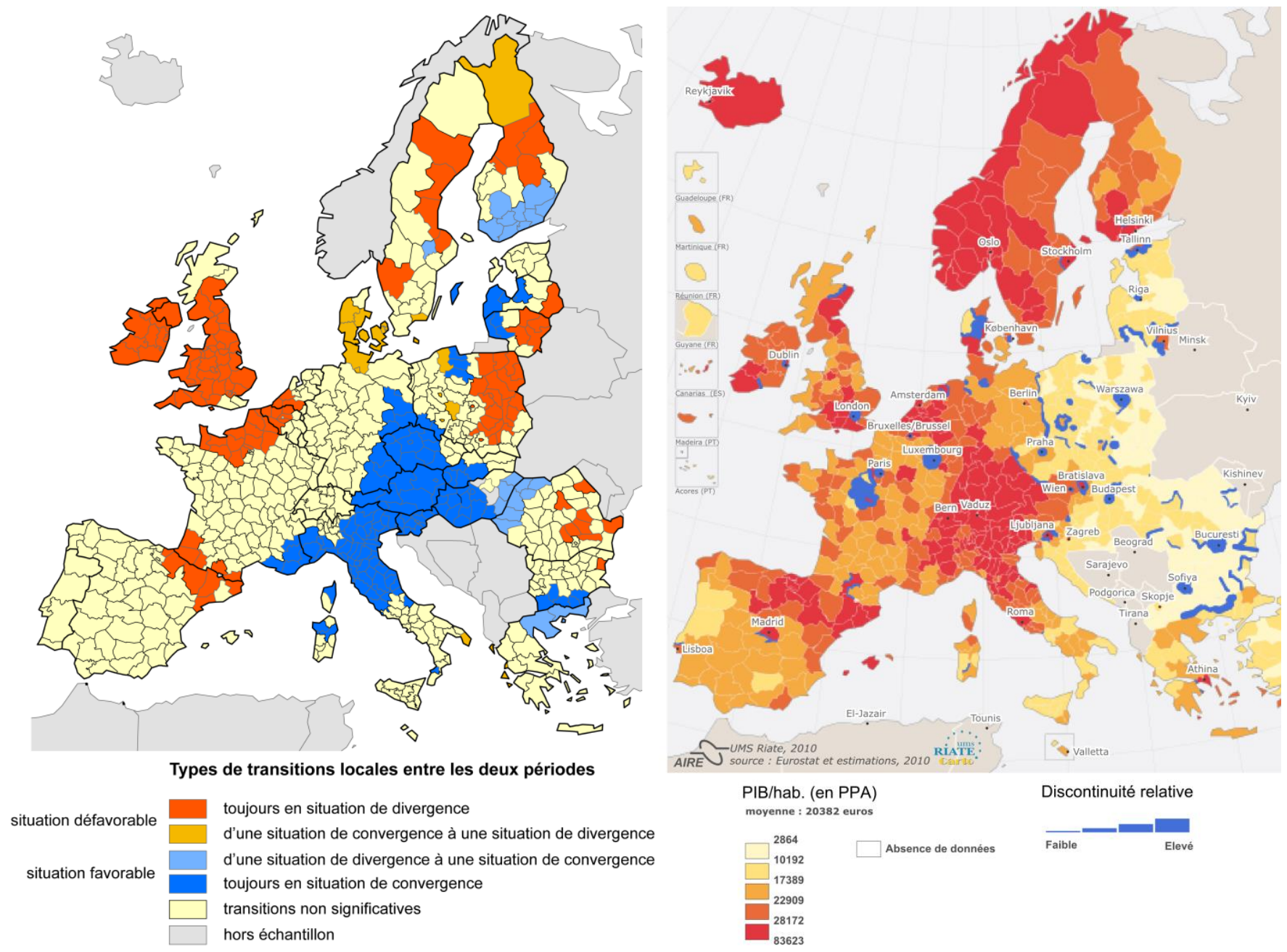

Dans le cas de l'UE, la variabilité des maillages ${ }^{3}$ est un obstacle à la comparabilité des territoires inégalement subdivisés. Une solution pour résoudre cette difficulté consiste à redistribuer les informations dans un carroyage régulier avant de les analyser ou de les visualiser (voir définition de «maillage», Pumain Hypergéo). On passe ainsi de représentations discontinues à des représentations continues de l'information. La différence de taille des mailles au niveau NUTS 2 est un problème. Claude Grasland et Malika Madelin (2006) préconisent, parmi les multiples solutions, d'utiliser un maillage intermédiaire entre le niveau NUTS 2 et le niveau NUTS 3 : le niveau NUTS 2/3. Les résultats de l'analyse sont donc présentés au niveau NUTS 2/3. Ce dernier permet d'avoir des unités territoriales de taille similaire et donc de dépasser, au moins en partie, le MAUP.

\footnotetext{
${ }^{3}$ Concernant l'adéquation entre les différents niveaux administratifs existant dans les États membres et les trois niveaux NUTS ainsi défini, il règne une importante hétérogénéité. En général, au sein de chaque pays, la structure régionale est composée de deux niveaux prépondérants (Länder et kreise en Allemagne, regioni et provincie en Italie, communidades autonomas et provencias en Espagne, régions et départements en France, etc.). A ces deux niveaux institutionnels nationaux correspondent des niveaux de NUTS différents : 1 et 3 pour l'Allemagne, 2 et 3 pour la France, 1 et 2 pour la Belgique. Enfin, en raison d'une aire ou d'une population trop réduite, certains pays ne disposent pas de tous les niveaux régionaux, c'est notamment le cas du Luxembourg ou du Danemark qui ne possèdent pas de régions NUTS 1 ou NUTS 2 et sont définis comme pouvant être de niveau 0,1 ou 2 .
} 


\subsection{Une convergence économique différenciée dans le temps et l'espace}

L'étude de la cartographie des indices locaux de convergence (carte 1) met en lumière une multipolarisation de la convergence. D'abord, le fait d'avoir retenu une portée de $300 \mathrm{~km}$ a permis de mettre en lumière les oppositions générales du territoire européen et d'éliminer les contrastes locaux. Il en résulte de grandes plages homogènes de convergence ou de divergence et ce, indépendamment des frontières. Ceci conforte l'hypothèse selon laquelle les inégalités économiques régionales sont par nature spatialement concentrées. De nombreuses régions de l'UE semblent former des « macro-clusters » de rattrapage économique ou au contraire de divergence. L'ouverture croissante des frontières liée à l'intégration des PECO dans l'UE a introduit des différenciations spatiales nouvelles qui ont profité en particulier aux régions occidentales des PECO. La carte 4 révèle la présence de fortes discontinuités spatiales dans les PECO et d'une manière plus générale entre les régions métropolitaines et leur hinterland. Les discontinuités observées ont un impact significatif sur le processus d'intégration régionale. Comme le soulignent Philippe De Boe, Claude Grasland et Adrian Healy (1999), les discontinuités peuvent être perçues de différentes façons. Soit elles peuvent être perçues comme génératrices de difficultés à l'intégration dans le sens où les différences de niveau économique peuvent réduire les possibilités de relations et d'interactions (cf. 2.2.). Soit elles peuvent être vues comme un potentiel pour accroître l'intégration dans le sens où le « différentiel » qu'elles apportent peut générer des flux entre les régions qui tendent à participer à la convergence locale et à l'homogénéisation interrégionale (cf. 2.3.).

Parmi les régions qui sont passées d'une situation de divergence à une situation de convergence locale, on distingue très nettement un groupe de régions s'étendant le long de la frontière hongro-roumaine (carte 1 et carte 3). Cette bande territoriale est marquée par une absence de discontinuités (carte 4) montrant ainsi l'intégration régionale à l'œuvre. Ces judete ${ }^{4}$ abritent quatre grandes villes que sont Satu Mare, Oradea, Arad et Timişoara. Ces dernières entretiennent des relations particulièrement étroites entre elles - via un trafic ferroviaire et routier dense - mais également avec la Hongrie. Cette zone étroite qui s'étend de la Yougoslavie à l'Ukraine est particulièrement attirée par l'Europe occidentale la remettant ainsi dans l'orbite de l'Europe centrale et germanique, dont elle fut un élément jusqu'en 1920 (Rey, 1996). Ce retour d'appartenance à la Mittleuropa a créé une situation de convergence économique locale. Cette zone s'est plus facilement ouverte au capitalisme occidental que d'autres régions roumaines (Cristescu, 2004). Les équipements commerciaux, les entreprises privées locales, étrangères et mixtes soulignent cette dynamique nouvelle. En outre, la présence d'une forte minorité hongroise et allemande dans cette zone facilite les relations que cette région de la Roumanie peut entretenir avec le reste de l'Europe. Le chômage y est moins fort que dans le reste de la Roumanie car d'autres activités sont venues se substituer très rapidement à la désindustrialisation engagée dans les années 1990. En outre, le secteur agricole y est plus performant qu'ailleurs en raison de la mise en place d'un système intensif combinant cultures céréalières et élevages bovins et en raison également d'une grande qualité du terroir du Banat. En revanche, la démographie est

\footnotetext{
${ }^{4}$ Unité administrative roumaine correspondant en France au département (NUTS 3).
} 
caractérisée par une stagnation de la population liée en partie à une baisse de la fécondité et à un vieillissement de la population particulièrement prononcés en Banat. Enfin, Timişoara connait depuis ces dix dernières années un dynamisme remarquable lié en partie à son éloignement à Bucarest mais aussi à son dynamisme universitaire avec les activités qui y sont liées (industrie électrotechnique et agro-alimentaire). Ainsi, les récentes mutations de cet espace frontalier concourent à la mise en place d'une convergence locale et d'une forme de cohésion territoriale localisée.

Parmi les questions que l'on peut se poser à la lecture des cartes 1 et 3 , il en est une qui mérite toute notre attention : est-ce que les régions qui étaient en situation de divergence locale sur la période 1995-2000 ont convergé à la fin de la période d'étude (2006) ? D'autres questions peuvent en découler : existe-t-il des régions ou un phénomène de divergence est toujours observable?

\subsection{Des " poches " de persistance de divergence locale...}

De nombreuses régions sont caractérisées par une persistance du phénomène de divergence locale entre les deux périodes d'analyse comme en Irlande et en Grande-Bretagne. Le " tigre celtique » des années 1990/2000 a enregistré des taux de croissance spectaculaires liés à la fois à une politique nationale de relance, un environnement fiscal et administratif favorable et à l'importance des Fonds de cohésion perçus (Murphy, 2000; Cornu et al., 2006). Or, les régions périphériques de Dublin ont enregistré un développement économique important mais sans commune mesure avec la région capitale. En outre, le phénomène de divergence locale observé en Angleterre s'explique par le poids écrasant de la région londonienne (région la plus prospère de toute l'UE). L'effet de diffusion de la croissance régionale des pôles dynamiques (ici la région de Dublin et de Londres) vers les régions périphériques en retard (voire en déclin pour les plus septentrionales d'entre elles) ne s'est pas ou peu opéré ; ou tout du moins, il n'a pas été suffisant pour permettre une convergence économique locale des régions de la zone considérée. Ceci se conforme à la théorie de Gunnar Myrdal (1957) qui considérait la croissance comme un processus spatial cumulatif susceptible d'accroître les disparités régionales et conduisant à une polarisation spatiale de l'économie (concentration de la richesse dans quelques pôles). Selon lui, les effets des économies d'agglomération conduisent à un processus cumulatif de croissance créant des différences entre régions et engendrant un cercle vicieux. Ainsi, il note qu'il existe une " causation circulaire cumulative » de la pauvreté : nulle force équilibrante ne vient corriger les déséquilibres économiques et sociaux. Ce processus de renforcement de la richesse ou de la pauvreté donne lieu à des effets de lessivage - «backward effect »(les régions attractives drainent vers elles les ressources aux dépens des autres régions) mais aussi à des effets de diffusion - «spillover effect» - de la croissance qui, eux, profitent aux régions périphériques. C'est l'observation empirique de la polarisation de l'économie qui pousse Myrdal à conclure que les effets de remous (forces centripètes) dominent finalement les effets de propagation (forces centrifuges). La poche de divergence locale identifiée dans les îles britanniques constitue un élément de validité de sa théorie. 
Dans le cas de la Roumanie, on observe une autre poche de divergence locale en périphérie de la capitale. Cela s'explique en partie par le poids écrasant de Bucarest vis-à-vis de ses régions voisines. En effet, alors que les IDE ont connu un décollage spectaculaire ${ }^{5}$, ces derniers se sont essentiellement concentrés dans la capitale. Bucarest possède ainsi $85 \%$ du PIB national, elle détient plus de $20 \%$ du volume de l'exportation nationale et presque $40 \%$ de l'importation, elle accumule $55 \%$ du PIB national des dépenses en R\&D et elle enregistre un taux de chômage inférieur de moitié à la moyenne nationale $(3,4 \%$ contre $7,2 \%$ pour la Roumanie en 2011). Bucarest concentre ainsi une part très importante des IDE créant des relations asymétriques entre la capitale et ses régions voisines (cf. carte 4). En outre, cette région possède un solde migratoire positif et attire particulièrement la population jeune (Rey et al., 2007). Tout ceci amène Octavian Groza et Violette Rey (2008) à dire que Bucarest est un des espaces d'Europe orientale « qui gagne ». L'augmentation des disparités économiques interrégionales caractérisée par la persistance de poches locales de divergence peut s'expliquer par le fait qu'un nombre limité de pôles de croissance concentrent une grande partie des capitaux, de la main d'œuvre et des investissements publics (notamment en termes d'infrastructures). Le fait que Bucarest ait un poids considérable vis-à-vis de son environnement régional fournit un exemple concret de la théorie de Paul Krugman (1996) sur l'effet « d'ombre d'agglomération ». Ce cas est observé lorsqu'il existe une domination importante d'un centre économique sur le reste de son territoire. Compte tenu des forces centripètes, les activités et les individus sont attirés par les grandes métropoles au détriment des régions alentours, suivant les prédictions du modèle gravitationnel. Dès lors, des pôles secondaires ne peuvent apparaître qu'à une distance suffisante pour échapper à ce phénomène d'attraction, laissant les espaces intermédiaires relativement vides.

\section{3. ... et la formation d'un club de convergence économique en Europe centrale}

Un nombre important de régions situées de part et d'autre de l'ex rideau de fer sont caractérisées par une situation de convergence économique locale malgré des niveaux de PIB/hab. très différents le long des frontières (cf. carte 2 et 4). De fait, le « mur de l'argent» (Grasland, 2004) qui avait succédé au « rideau de fer » semble progressivement se fissurer à son tour. Ces régions enregistrent, entre les deux périodes d'analyse, une forte convergence et peuvent constituer, de part leur regroupement géographique, un club de convergence économique.

Si les régions de Bohême et de Moravie de la République tchèque convergent avec leurs voisines, c'est parce que les deux provinces partagent un niveau de développement similaire issu de la première révolution industrielle $^{6}$ mais aussi parce que le réseau de petites villes et villes moyennes est dense sur tout le territoire $^{7}$. La République tchèque et la partie occidentale de la Slovaquie enregistrent des taux de chômage

\footnotetext{
${ }^{5}$ Augmentation de $41 \%$ en 2006 par rapport à 2005, soit en 2006 l'équivalent de 8,6 milliards d'IDE.

${ }^{6}$ Notons que ces provinces étaient, au début du XXème siècle, le centre économique de l'empire des Habsbourg.

${ }^{7}$ La densité moyenne est élevée (130 hab./ $\left.\mathrm{km}^{2}\right)$ et peu contrastée dans le pays. De ce point de vue, les pays tchèques ont offert à l'idéologie communiste de l'égalitarisation spatiale et de la suppression des oppositions ville-campagne une trame prête.
} 
bas du fait des IDE effectués en particulier par le marché allemand. Le principal facteur explicatif de l'arrivée de ces IDE est sans conteste l'émergence d'un marché de consommateurs (Bourdin et al., 2009 ; Lepesant, 2010). La présence d'une main-d'œuvre qualifiée ${ }^{8}$ et à bas coût explique également cela. Cependant, il faut noter que l'intensité de ces investissements n'est pas la même partout. En effet, l'ouverture croissante des frontières liée à l'intégration des PECO dans l'UE a introduit des différenciations spatiales nouvelles qui ont profité en particulier à la capitale tchèque. Le processus de privatisation des grandes entreprises est important à Prague, en Bohême occidentale et au contact de la Bavière alors qu'il est davantage boudé prêt des frontières polonaises et slovaques. Néanmoins, la région silésienne tchèque crée une sorte de contrepoids à la capitale avec la ville d'Ostrava (320 000 hab.) permettant de faciliter les synergies de conversion économique et de développement d'innovations avec les régions slovaques situées de l'autre côté de la frontière.

La convergence économique locale observée dans la partie Sud-Ouest de la Slovaquie s'explique par le dynamisme incontesté de Bratislava qui a su mettre en avant sa position géographique comme une ville de passage et de transit en valorisant le contact danubien. Sur ce cinquième de territoire sont réunies les agricultures et les industries les plus performantes du pays. La métropole échappe au chômage et concentre près de $60 \%$ du total des IDE reçu par la Slovaquie. Ce décrochement de la capitale vis-à-vis du reste du pays s'explique également par son histoire. Bratislava a dû se construire une identité de capitale politique de plein exercice depuis 1993 en mettant en avant la nécessité d'une métropole avec une véritable personnalité slovaque. Elle a dû relever le défi postindustriel et postsoviétique tout en retrouvant sa tradition danubienne cosmopolite. Cette convergence économique locale entre la région de Bratislava et les régions voisines plus à l'Ouest ne doit pas faire oublier le risque de l'isolement des quatre autres cinquièmes du territoire slovaque. S'agissant de la situation de la Hongrie, on observe une légère dissymétrie entre l'Ouest compris dans la large poche de convergence locale et l'Est situé en partie à l'écart de ce phénomène de rattrapage (carte 3 et 4). En termes d'IDE, la Hongrie occidentale est la référence de par son attractivité liée en partie à sa position géographique mais aussi due à son choix de miser sur une privatisation ouverte au capital international. Ces IDE ont été effectués pour une grande part dans le domaine des télécommunications, dans les branches commerciales et de service participant à la tertiarisation et à la reconversion rapide de son économie. La Hongrie inspire une relative stabilité politique et la main d'œuvre qualifiée et flexible sont des facteurs supplémentaires ayant favorisé les IDE.

Nous l'avons vu, les phénomènes de convergence économique globale peuvent masquer des divergences locales. Les situations sont multiples et trouvent souvent leurs origines dans un passé plus ou moins lointain mais s'expliquent aussi de par leur localisation géographique. Ainsi, la proximité géographique des régions

\footnotetext{
${ }^{8}$ Un quart du salaire moyen allemand et 13 à $40 \%$ de moins qu'en Hongrie ou en Pologne.
} 
centre-orientales à l'Europe des Quinze constitue un facteur de percolations financières, techniques, touristiques, d'emplois et de commerce.

\section{Conclusion}

La prise en compte de l'importance de la dimension spatiale dans l'étude de la convergence économique permet d'appréhender différemment les inégalités économiques régionales dans l'Union européenne. La cartographie des résultats de l'indice proposé montre la présence simultanée d'un processus de convergence et de divergence économique locale reposant souvent sur des avantages dits « initiaux » que possèdent certaines régions. Leur croissance plus rapide s'explique en partie par leur localisation (dissymétrie Ouest/Est), leur passé (histoire récente et plus longue) et leur dotation en équipements (liés essentiellement à des investissements nationaux et européens). L’hétérogénéité de l'espace géographique apparaît alors comme un élément explicatif et constitutif des disparités régionales de développement mis en évidence notamment par l'ouverture du pays à l'Ouest et le démantèlement du CAEM (Bourdin, 2011). Les deux derniers élargissements de l'UE à l'Est ont à la fois encouragé la croissance dans ces nouveaux Étatsmembres mais ont créé également un décalage croissant au niveau régional. Les régions les plus éloignées $\mathrm{du}$ « coeur de l'Europe » voient leur retard s'accentuer, y compris par rapport à des régions plus à l'ouest au sein d'un même pays. Comme le souligne Michel Sollogoub (2006), le rattrapage économique des nouveaux membres de l'Union risque donc d'être inégal en fonction des pays et des régions et même assez problématique pour les pays et régions les plus à l'Est et les plus éloignées du cœur européen, qui auront beaucoup de difficultés à accélérer leur croissance et leur compétitivité.

La persistance de divergences locales ou au contraire l'apparition de clubs de convergence appellent des réponses politiques différenciées. Ces dernières doivent tenir compte des différents facteurs à l'origine de la convergence économique des régions parmi lesquels on retrouve les Fonds structurels européens, les effets d'héritages et de contexte ainsi que les effets de proximité. Devant l'incapacité de la politique de cohésion à réduire les disparités interrégionales au sein de chaque pays, notre étude sur la convergence économique locale permet d'apporter des éléments de réflexion sur la manière de repenser la future politique régionale européenne après 2013 afin de la rendre plus efficace. La prise en compte de la localisation géographique et de la structure du voisinage de chacune des régions européennes dans la manière de concevoir la politique de cohésion peut constituer une piste à explorer. Nos travaux confirment ainsi ceux de Philippe Montfort, Sandy Dall'Erba, Julie Le Gallo ou encore Cem Ertur et Wilfried Koch sur la nécessité de prendre en compte les effets de voisinage dans la politique régionale de convergence. Certaines régions considérées comme des périphéries marginalisées du fait de leur localisation géographique et du fait qu'elles sont caractérisées par une situation de divergence locale mériteraient dès lors un traitement spécifique en termes d'aides européennes. A ce sujet, parmi les extensions futures de ces travaux, il serait intéressant d'ajouter les 
régions des pays voisins de l'UE (Turquie, Ukraine, Moldavie, Belarus, Russie) pour analyser valablement les phénomènes de convergence/divergence aux limites orientales de l’UE.

\section{Bibliographie}

Abreu M., de Groot H., Florax R. (2005). «Space and Growth: A Survey of Empirical Evidence and Methods ». Région et Développement, $\mathrm{n}^{\circ} 21, \mathrm{p} .12-43$

Arbia G., Le Gallo J. et Piras G. (2008). « Does evidence on regional economic convergence depend on the estimation strategy? Outcomes from analysis of a set of NUTS2 regions ». Spatial Economic Analysis, vol. 3, p. 209-224

Barro R. J. et Sala-i-Martin X. (1992). «Convergence », Journal of Political Economy, vol. 100, n 2, p. 223-51

Barro R.J. et Sala-I-Martin X. (1995). Economic growth theory. Boston : McGraw-Hill, 672 p.

Baumol W.J. (1986). "Productivity growth, convergence and welfare : what the long run data show ». American Economic Review, vol. 76, p. 1072-1085

Baumont C. (1998). Économie géographique et intégration régionale : Quels enseignements pour les Pays $d^{\prime}$ Europe Centrale et Orientale? LATEC, document de travail - Economie, ${ }^{\circ} 11$

Beine M., Docquier F. et Hecq A. (1999). Convergence des groupes en Europe : une analyse sur données régionales. ULB Institutional Repository, document de travail, n²013/10459

Bernard A. et Durlauf S. (1995) "Convergence in international output ». Journal of Applied Econometrics, vol. 10, p. $97-108$

Bivand R. et Brunstad R. (2003). "Regional growth in Western Europe: an empirical exploration of interactions with agriculture and agricultural policy ». In Fingleton B. (dir.), European Regional Growth. ed. Berlin : Springer, p. 351-373

Bivand R. et Brunstad R. (2005). «Further explorations of interactions between agricultural policy and regional growth in Western Europe - approaches to nonstationarity in spatial econometrics ». 49ème Congrès de l'ERSA, Vienne, Autriche 2 au 6 août 2005

Bourdin S., Le Thiec A. et Elissalde B. (2009). " Le changement spatial dans l'industrie automobile en Europe centrale et orientale : entre effets d'héritage, cycles de vie et transition ». Géocarrefour, vol. 84, ${ }^{\circ} 3$, p. 193-204

Bourdin S. (2011). «Repenser le futur de la politique de cohésion européenne pour les pays d'Europe centrale et orientale : la prise en compte de l'espace géographique comme piste de recherche ». Bulletin de la Société Géographique de Liège, n55, p. 43-59

Carlino G.A. et Mills L. (1996). «Testing neoclassical convergence in regional incomes and earnings ». Regional Science and Urban Economics, vol. 26, n 6 , p. 565-590

Chaterrji J. (1992). «Convergence clubs and endogeneous growth ». Oxford Review of Economic Policy, vol. 8, p. 57-69

Choi C. Y. (2004). "A Reexamination of Output Convergence in the U.S. States: Toward Which Level(s) are they Converging? ». Journal of Regional Science, vol. 44, n 4, p. 713-741 
Cornu G., Bailly G., Merceron J-C. et Reiner, D. (2006). Irlande : quel avenir pour le tigre celtique ?. Fait au nom de la commission des affaires économiques du Sénat, $n^{\circ} 204,42$ p.

Cristescu J. (2004). «Regional restructuring in Romania, a relevant agent of change ? », European Spatial Research and Policy, vol. 11, n², p. 105-117

Dall'Erba S. (2004). Productivity Convergence and Spatial Dependence Among Spanish Regions. REAL, Université de l'Illinois à Urbana-Champaign, document de travail, nº 04-T-03

Dall'Erba S. et Le Gallo J. (2008). «Regional convergence and the impact of European structural funds over 1989-1999: a spatial econometric analysis ». Papers in Regional Science, vol. 87, p. 219-244

De Boe P., Grasland C. et Healy A. (1999) « Spatial integration ». Strand 1.4 In Study Programme on European Spatial planning

De Siano R. and D’Uva M. (2006). « Club convergence in European regions ». Applied Economic Letters, vol. $13, n^{\circ} 9$, p. $569-574$

Eckey H-F., Kosfeld R. et Türck M. (2007). «Regional Convergence in Germany: a Geographically Weighted Regression Approach ». Spatial Economic Analysis, vol. 2, n 1, p.45-64

Ertur C. et Koch W. (2005). «Une analyse exploratoire des disparités régionales dans l'Europe élargie ». Région et développement, vol. 21, p. 65-92.

Ertur C. et Le Gallo J. (2009). «Regional growth and convergence : heterogenous reaction versus interaction in spatial economic approaches ». In Capello R. et Nijkamp (dir.), Handbook of regional growth and development theories, New-York : Edward Elgar, p. 705-730

Ertur C., Le Gallo J. et Lesage J. (2007). «Local versus global convergence in Europe: a Bayesian spatial econometric approach ». Review of Regional Studies, vol. 37, p. 82-108.

EUROSTAT (2007). Le produit intérieur brut régional dans l'Union européenne en 2004. Office des publications officielles des Communautés européennes, Luxembourg

EUROSTAT (2009). Les chiffres clés de l'Europe. Office des publications officielles des Communautés européennes, Luxembourg

EUROSTAT (2010). Produit intérieur Brut, Annuaire régional d'Eurostat 2010. Office des publications officielles des Communautés européennes, Luxembourg

Evans P. et Karras G. (1996). «Convergence Revisited ». Journal of Monetary Economics, vol. 37, p. 249265

Fagerberg J. et Verspagen B. (1996). «Heading for Divergence? Regional Growth in Europe Reconsidered ». Journal of Common Market Studies, vol. 34, n 3, p. 431-448

Fingleton, B et Lopez-Bazo E (2006). «Empirical growth models with spatial effects ». Papers in Regional Science, vol. 85, p.177-198

Fuss C. (1999). «Mesures et tests de convergence : une revue de littérature ». Revue de l'OFCE, vol. 74, $\mathrm{n}^{\circ} 1$, p. $159-179$

Galor O. (1996). «Convergence ? inferences from theorical models ». The Economic Journal, $\mathrm{n}^{\circ} 106, \mathrm{p}$. 1056-1069 
Grasland C. (2004). "Les inégalités régionales dans une Europe élargie ». In Chavance B. (dir.). Les incertitudes du grand élargissement : L'Europe centrale et balte dans l'intégration européenne. Paris: L'Harmattan, $284 \mathrm{p}$.

Grasland C. et Madelin M. (dir.) (2007). The modifiable area unit problem. Final report of Espon Project 3.4.1., ESPON

Groza O. et Rey V. (2008). " Bulgarie et Roumanie, une affaire d'européanisation », L'Espace géographique, $\mathrm{n}^{\circ} 4$, Tome 37, p. 289-296

Getis A. (2009). « Spatial Weights Matrices ». Geographical Analysis, vol. 41, n 4, p. 404-410

Hall S., Robertson D. et Wickens M. R. (1997). «Measuring Economic Convergence ». International Journal of Finance \& Economics, vol. 2, n 2, p. 131-43

Henin P.-Y. et Le Pen Y. (1995). «Les épisodes de la convergence européenne », Revue économique, vol. $46, n^{\circ} 3$, p. $667-677$

Islam N. (2003). « What have we learnt from the convergence debate ». Journal of Economic Surveys, vol. 17, p. 309-362

Janikas M.V. et Rey S. J. (2005). Spatial clustering, inequality and income convergence. Urban/Regional Economics, document de travail, $n^{\circ} 0501002$

Janikas M.V. et Rey S.J. (2008). «On The Relationships Between Spatial Clustering, Inequality, And Economic Growth In The United States : 1969-2000 ». Région et Développement, vol. 27, p. 13-34

Le Gallo J. et Dall'erba S. (2004). «Evaluating the Temporal and the Spatial Heterogeneity of the European Convergence Process, 1980-1999 ». Journal of Regional Science, vol. 46, n², pp. 269-288

Le Gallo J., Dall'Erba S. et Guillain R. (2011). «The Local versus Global Dilemma of the Effects of Structural Funds ». Growth and Change, vol. 42, n 4, p. 466-490

Le Pen Y. (2011). «A pair-wise approach to output convergence between European regions ». Economic Modelling, vol. 28, n 3, p. 955-964

Lepesant G. (2010). Géographie économique de l'Europe centrale : recomposition et européanisation des territoires. Paris : Les presses de Sciences Po, 352 p.

Mankiw N.G., Romer D., Weil D.N. (1992). «A contribution to the empirics of economic growth ». Quarterly Journal of Economics, ${ }^{\circ} 107$, p. 407-437

Montfort P. (2008). «Convergence of EU régions. Measures and Evolution ». Bruxelles : DG Politique Régionale, document de travail, $\mathrm{n}^{\circ} 1,20 \mathrm{p}$.

Murphy A. (2000). The Celtic Tiger : an analysis of Ireland's economic growth performance. European University Institute, Robert Schuman Centre, document de travail, $\mathrm{n}^{\circ} 16$

Neven D. et Gouyette C. (1994). «European Integration and Regional Growth ». Revue économique, $\mathrm{n}^{\circ} 3, \mathrm{p}$. 703-713

Parlement européen (2007). Les disparités régionales et la cohésion : quelles stratégies pour l'avenir ?. Bruxelles, 151 p.

Quah D. (1993). "Empirical cross-section dynamics in economic growth ». European Economic Review, n³7, p. 426-443 
Quah D. (1996). «Regional convergence clusters across Europe ». European Economic Review, vol. 40, p. $143-156$

Quah D. et Durlauf S.N. (1998). The new empirics of economic growth. Wisconsin Madison, Social Systems, document de travail, $\mathrm{n}^{\circ} 8$

Quah D. (1999). Cross-country growth comparison : theory to empirics. CEPR, document de travail, $\mathrm{n}^{\circ} 2294$

Rey V. (1996). Europes orientales, Russie, Asie centrale. In Brunet R., Dollfus O. (dir.), Géographie Universelle, tome 10. Paris-Montpellier : Hachette-Reclus, 551 p.

Rey V., Groza O., Ianos I et Patroescu M. (2007). Atlas de la Roumanie. Montpellier, Paris : CNRS GDRE S4-La Documentation française, coll. «Dynamique du territoire », $211 \mathrm{p}$.

Rey S.J. et Le Gallo J. (2009). « Spatial analysis of economic convergence ». In Mills T.C. et Patterson K. (eds.). The Palgrave Handbook of Econometrics, Volume II: Applied Econometrics, New-York : Palgrave McMillan, $1424 \mathrm{p}$.

Rogerson P.A. (2010). Statistical methods for geography. A student's guide. Londres : Sage, 368 p.

Sapir J., Aghion P., Bertola G., Hellwig M., Pisani-Ferry J., Rosati D., Viñals J., Wallace H., Buti M., Nava M. et Smith P.M. (2004). An Agenda for a Growing Europe: The Sapir Report. Oxford : Oxford University Press, $236 \mathrm{p}$.

Sollogoub T. (2006). «Quelle convergence au sein de l'Union européenne élargie : Les enjeux pour la Bulgarie et la Roumanie ? ». Questions d'Europe, Fondation Robert Schuman, nº41

Solow R.M. (1956). «A contribution to the theory of economic growth ». Quaterly Journal of Economics, 70, p. 65-94

Toral A. (2002). « Measuring distance in the Spanish Regional Convergence Process ». Revue d'Économie Régionale et Urbaine, n5, p. 789-804

Virol S. (2006). «Distance temps, discontinuité des interactions spatiales et concentration globale de l'espace européen ». Revue d'Économie Régionale et Urbaine, n¹, p. 7-26

Williamson J. G. (1965). «Regional Inequality and the Process of National Development: A Description of the Patterns ». Economic Development and Cultural Change, n ${ }^{\circ}$ 13, p. 1-84

Yildirim J., Öcal N. et Özyildirim S. (2009). « Income Inequality and Economic Convergence in Turkey: A Spatial Effect Analysis ». International Regional Science Review, vol. 32, n 2, p. 221-254

Yu D-L. (2006). « Spatially varying development mechanisms in the Greater Beijing Area: a geographically weighted regression investigation ». The Annals of Regional Science, vol. 40, n 1, p. 173-190 